\title{
Artigo/Article
}

\section{A lesão apical em cardiopatas chagásicos crônicos: estudo necroscópico}

\author{
Apical lesions in Chagas' heart disease patients: an autopsy study
}

\section{Cristina Brandt Friedrich Martin Gurgel ${ }^{1,2,3}$, Maria Cristina Furian Ferreira ${ }^{4}$, Clayde Regina Mendes ${ }^{5}$, Elaine Coutinho ${ }^{4}$, Priscila Favoritto ${ }^{6}$ e Fernanda Carneiro ${ }^{6}$}

\section{RESUMO}

Introdução: A lesão apical ventricular é típica da cardiopatia chagásica e sua presença representa risco de fenômenos tromboembólicos. O objetivo deste trabalho é avaliar a frequência de LA à necropsia de portadores de cardiopatia chagásica crônica. Métodos: Análise retrospectiva de necropsias de chagásicos maiores que 17 anos. Efetuada análise estatística comparativa das variáveis clínicas e dos achados necroscópicos entre o grupo A (com lesão apical) e o grupo B (ausência de lesão apical). Resultados: Estudados 51 casos: 25 no grupo A (idade média de 53 anos, 64\% do sexo masculino)e 26 no B.. A LA localizava-se no ventrículo esquerdo em $80 \%$ casos. No grupo B, a média de idade foi de 56 anos e 46,1\% eram do sexo masculino. A forma clínica prevalente nos dois grupos foi a miopática, mas arritmia cardíaca também esteve presente em ambos (57,9\% no grupo A e 32,1\% no B). Foi constatada a presença de trombos em $60 \%$ dos casos do grupo A (53,3\% localizados na LA ) e 30,7\% no B; Conclusões: Houve predomínio da forma miopática nos casos com LA, com média de peso cardíaco maior em relação ao B. Em ambos os grupos observamos relação diretamente proporcional entre maior peso cardíaco e presença de tromboses. Houve predomínio do número de tromboses no grupo A, mais de 50\% eram localizadas na lesão, cujo diferencial clínico principal consistiu na presença maior de arritmias. A miopatia (com aumento de peso acima de $500 \mathrm{~g}$ ) foi primordial para aparecimento de tromboses.

Palavras-chaves: Miocardiopatia chagásica. Autópsia. Aneurisma cardíaco.

\begin{abstract}
Introduction: The presence of an apical ventricular lesion increases the risk of intracardiac thrombosis and thromboembolic phenomena. The study evaluated the incidence of apical lesions and intracardiac thrombosis in Chagas' heart disease patients at autopsy. Methods: A retrospective review of autopsies of Chagas' heart disease patients was conducted. Statistical analysis included comparison of clinical variables and autopsy findings between two groups: group A (apical lesions) and group B (no apical lesions). Results: A total of 51 cases of Chagas' disease patients were studied: 25 in group A (mean age 53 years-old; 64\% male) and 26 in group B. Apical lesions were verified in the left ventricle in $80 \%$ of cases. The prevalent clinical subtype in both groups was myopathic, but significant cardiac arrhythmia was present in $57.9 \%$ of patients in group A, while $76.9 \%$ in group B did not present arrhythmias. Mean heart weight was $500.9 \mathrm{~g}$ in group A and $408.4 \mathrm{~g}$ in group B. The presence of thrombosis occurred in $60 \%$ of group A with 8 (53.3\%) thrombi occurring in the apical lesion. Conclusions: The myopathic subtype was the most common clinical form in group $A$ and the mean heart weight was statistically higher in this group. Clear prevalence of thrombosis was verified in group A, with $50 \%$ located in the apical lesion, whose main differential factor was a greater incidence of arrhythmias. Myopathy (heart weight above $500 \mathrm{~g}$ ) was primordial for the presence of thrombosis in both groups.
\end{abstract}

Key-words: Apical lesion. Chagas' myocardiopathy. Autopsy. Heart aneurysm.

1. Disciplina de Semiologia Prática, Centro de Ciências da Vida, Faculdade de Medicina, Pontifícia Universidade Católica, Campinas, SP. 2. Grupo de Estudos da Doença de Chagas, Pontifícia Universidade Católica, Campinas, SP. 3. Ambulatório de Cardiologia, Hospital e Maternidade Celso Pierro, Pontifícia Universidade Católica, Campinas, SP. 4. Disciplina de Anatomia Patológica, Centro de Ciências da Vida, Faculdade de Medicina, Pontifícia Universidade Católica, Campinas, SP. 5. Disciplina de Estatística, Faculdade de Matemática, Centro de Ciências Exatas, Ambientais e Tecnologias, Pontifícia Universidade Católica, Campinas, SP. 6. Curso de Medicina, Pontifícia Universidade Católica, Campinas, SP. Endereço para correspondência: Dra. Cristina Brandt Friedrich Martin Gurgel. Rua MMDC 47/101, 13025-130 Campinas, SP.

Tel: $55193252-7600$

e-mail: cbfmgurgel@ig.com.br

Recebido para publicação em 19/04/2010

Aceito em 27/07/2010

\section{INTRODUÇÃO}

A doença de Chagas é uma antropozoonose de evolução crônica causada pelo Trypanosoma cruzi. No Brasil, estima-se que cerca de 3 milhões de pessoas estejam infectadas ${ }^{1,2}$.

A maioria dos pacientes alberga o parasito nos tecidos e sangue durante toda a vida, podendo ou não manifestar a doença, classificada em aguda e crônica, de acordo com o tempo de infestação e apresentação clínica. As formas crônicas mais corriqueiras são a digestiva e cardíaca; as mistas e o acometimento nervoso são menos comuns. O envolvimento cardíaco é a manifestação mais grave da doença, desenvolvendo-se em 25-30\% dos indivíduos infectados e pode levar a alterações do ritmo, fenômenos tromboembólicos e insuficiência cardíaca congestiva ${ }^{2}$.

Uma das manifestações típicas da cardiopatia chagásica crônica (CCC) é a lesão apical (LA), também denominada lesão vorticilar, aneurisma ventricular ou adelgaçamento de ponta, caracterizada por um variado grau de afilamento das fibras e eventual protusão da ponta do ventrículo esquerdo. Sob o ponto de vista anatomopatológico podem ser identificados dois tipos de lesão apical: por afastamento dos feixes musculares que formam o vórtex e por adelgaçamento progressivo da musculatura em direção ao seu vórtex (cornos). Os dois tipos diferem entre si em alguns aspectos morfológicos, como diâmetro (maior no primeiro) e espessura, maior quando não há afastamento dos feixes. Além disso, a trombose é mais frequente e a fibrose miocárdica é mais extensa nas lesões com adelgaçamento progressivo dos feixes musculares, sem afastamento dos mesmos ${ }^{3}$. Várias formas são descritas de lesão apical: em dedo de luva, mamilar, em raquete e quando denominada aneurisma, pressupõe-se que haja também um abaulamento do contorno externo cardíaco ${ }^{4}$.

Sua patogênese é controversa. Acredita-se que haja um caráter multifatorial e que mantenha relação com peculiaridades anatomofuncionais normais do ventrículo esquerdo, além de alterações cardíacas determinadas pela tripanosomíase. As possíveis 
etiopatogenias envolvem isquemia, inflamação, trombose, fatores mecânicos, espessura afilada da parede e alterações da cinética regional do ventrículo, relacionados ou não com a denervação parassimpática e/ou lesões diretas do sistema excito-condutor ${ }^{5-8}$.

O objetivo do presente estudo foi avaliar a frequência de lesão apical em chagásicos crônicos necropsiados e a presença de trombose intracardíaca em seus portadores.

\section{MÉTODOS}

Foi realizado levantamento retrospectivo de necropsias de pacientes chagásicos crônicos maiores que 17 anos realizadas no Departamento de Anatomia Patológica do Hospital e Maternidade Celso Pierro no período de 1982 a 2001. As necropsias ocorreram rotineiramente em todos os casos.

Para o diagnóstico da doença de Chagas, considerou-se, além’ da epidemiologia fortemente positiva, a positividade de pelo menos duas reações sorológicas diferentes realizadas em vida (fixação de complemento com título superior a $1 / 4$, e/ou imunofluorescência indireta com título igual ou superior a $1 / 40$, e/ ou hemaglutinação passiva com título igual ou superior a 1/32, e/ ou ELISA reagente). Foram excluídos do estudo todos os casos que apresentassem achados necroscópicos que pudessem influenciar nos resultados tais como valvopatias, cardiomiopatia hipertrófica, cardiopatia isquêmica, amiloidose e demais doenças de depósito.

A partir da avaliação dos prontuários, foram coletados dados gerais como sexo, idade e manifestações clínicas da doença de Chagas, especialmente cardíacas. Sob o ponto de vista anatomopatológico, a cardiopatia foi classificada em miopática (quando constatado no prontuário registro em vida de sintomas e sinais compatíveis com insuficiência cardíaca), dromopática (quando constatados distúrbios de condução), batmopática (quando evidenciados distúrbios do automatismo) e mista (quando coexistiam duas ou mais apresentações dentre as descritas) $)^{9}$. Foram avaliadas, adicionalmente, informações sobre o quadro clínico final e presença de co-morbidades.

Os corações foram estudados após fixação em formalina e cortados interessando o plano de 4 câmaras: átrios, valvas atrioventriculares, ventrículos e apex do VE. Os critérios anátomopatologicos utilizados foram os mesmos já descritos como elucidativos para o diagnóstico de LA. Não utilizamos a palavra aneurisma, pois em nenhum dos casos foi constatado abaulamento localizado na silhueta externa do coração.

Além da presença da LA e sua localização, foram analisadas as seguintes variáveis, descritas no relatório necroscópico: presença e localização de trombose intracardíaca, peso cardíaco em gramas e presença de embolia e/ou infarto e avaliação da causa mortis (presença de compressão bulbar por herniação das amídalas cerebelares, arritmia e choque de diversas etiologias).

Coletados os dados, foi realizada análise estatística comparativa das variáveis clínicas e dos achados necroscópicos considerando dois grupos distintos: grupo A (com lesão apical) e grupo B (sem lesão apical). Para análise estatística foi realizada a média aritmética e o desvio padrão para cada uma das variáveis quantitativas que necessitaram de seu cálculo ${ }^{10}$. Para análise inferencial foram utilizados os testes $t$ de Student e o teste qui-quadrado, considerando-se $\alpha=0,05$ para nível de significância ${ }^{11}$.

\section{Ética}

A análise dos prontuários foi aprovada pelo Comitê de Ética e Pesquisa da PUC-CAMPINAS, sob o protocolo 730/06.

\section{RESULTADOS}

No presente estudo, foram incluídas 51 necrópsias de pacientes cardiopatas chagásicos, sendo que 25 (49\%) foram classificados no grupo A e 26 (51\%) no B. Entre os grupos, não houve diferença significativa quanto ao sexo $\left[\chi^{2}(1)=0,543\right.$ e $\left.p>0,05\right]$. Observouse a ausência de lesão em $57,1 \%$ dos casos (12 pacientes) do sexo feminino e em $46,7 \%$ dos pacientes masculinos. No grupo A, a idade média foi de 52,84 anos (mínima-22, máxima-74, \pm 12,96 anos) e no B foi de 56,42 anos (mínima-17, máxima-84, $\pm 17,88$ anos); o teste $t$ revelou que não há diferença significativa da idade entre os grupos $[t$ $(49)=0,822 ; p>0,05]$. Contudo, observou-se que no grupo A houve predomínio de pacientes na faixa etária entre 50 e 59 anos (40\%, 10 pacientes), enquanto no grupo B eles concentravam-se na faixa etária de 60 a 69 anos (10 pacientes - 38,5\%) (Figura 1).

Quanto ao quadro clínico, a insuficiência cardíaca congestiva (ICC) esteve presente em 13 (52\%) pacientes com lesão apical e em 12 pacientes sem lesão (48\%), não havendo, contudo, diferença estatisticamente significativa entre os grupos $\left[\chi^{2}(1)=0,174\right.$ e $p$ > $0,05]$. Entretanto, em relação à arritmia cardíaca (AC), 57,9\% (22 pacientes) dos pacientes no grupo A tiveram arritmias e 76,9\% (10 pacientes) do grupo B não as apresentaram $\left[\chi^{2}(1)=4,699\right.$ e $p<$ 0,05] (Tabela 1).

A co-morbidade mais frequente nos dois grupos foi a hipertensão arterial sistêmica, constatada em 7 (28\%) e em 11 (42,3\%) indivíduos pertencentes aos grupos A e B respectivamente. Não houve diferença estatisticamente significativa $\left[\chi^{2}(1)=2,092\right.$ e $\left.\mathrm{p}>0,05\right]$ entre os grupos em relação a esta co-morbidade. Também, não foi significativa a variação da frequência de trombose entre os grupos $\left[\chi^{2}(1)=1,000\right.$ e $p>0,05]$. Contudo, nos 25 pacientes com lesão apical, 15 tiveram trombose. Desses, 7 (46,7\%) tiveram trombose na lesão. Nos 26 sem lesão, apenas 3 tiveram trombose.

Analisando-se os casos do grupo A em que se constatou trombose, verificou-se que $80 \%$ dos corações tinham peso entre $500-599 \mathrm{~g}$. Nos casos em que o peso estava acima de $600 \mathrm{~g}, 100 \%$ tinham trombose. No grupo B, a trombose esteve presente em $8(30,7 \%)$, localizados principalmente no ventrículo esquerdo $\left(\mathrm{n}^{\circ}=5\right.$, $62,5 \%)$. Neste grupo, todos os pacientes com peso cardíaco acima de $500 \mathrm{~g}\left(\mathrm{n}^{\mathrm{o}}=3\right)$ possuíam trombo intracardíaco. No grupo $\mathrm{A}$, ocorreu tromboembolismo pulmonar em $8(53,3 \%)$ casos, sendo que em $100 \%$ havia trombose em átrio direito e, em um caso, na lesão apical do ventrículo direito. No grupo B, encontramos 5 (19,2\%) casos de tromboembolismo pulmonar, com 3 (60\%) trombos localizados em átrio direito (Tabela 2). Não houve diferença significativa em relação ao peso cardíaco, quando comparamos os pacientes com e os pacientes sem trombose $[t(40)=1,339 ; \mathrm{p}>0,05]$ (Figura 2).

Embora não tenha sido observada diferença estatisticamente significativa $\left[\chi^{2}(1)=3,229\right.$ e $\left.p>0,05\right]$, entre os pacientes com lesão apical, o óbito foi atribuído a distúrbio do ritmo em 21 (84\%), enquanto no grupo B este foi a causa de óbito em 15 (46,1\%). 


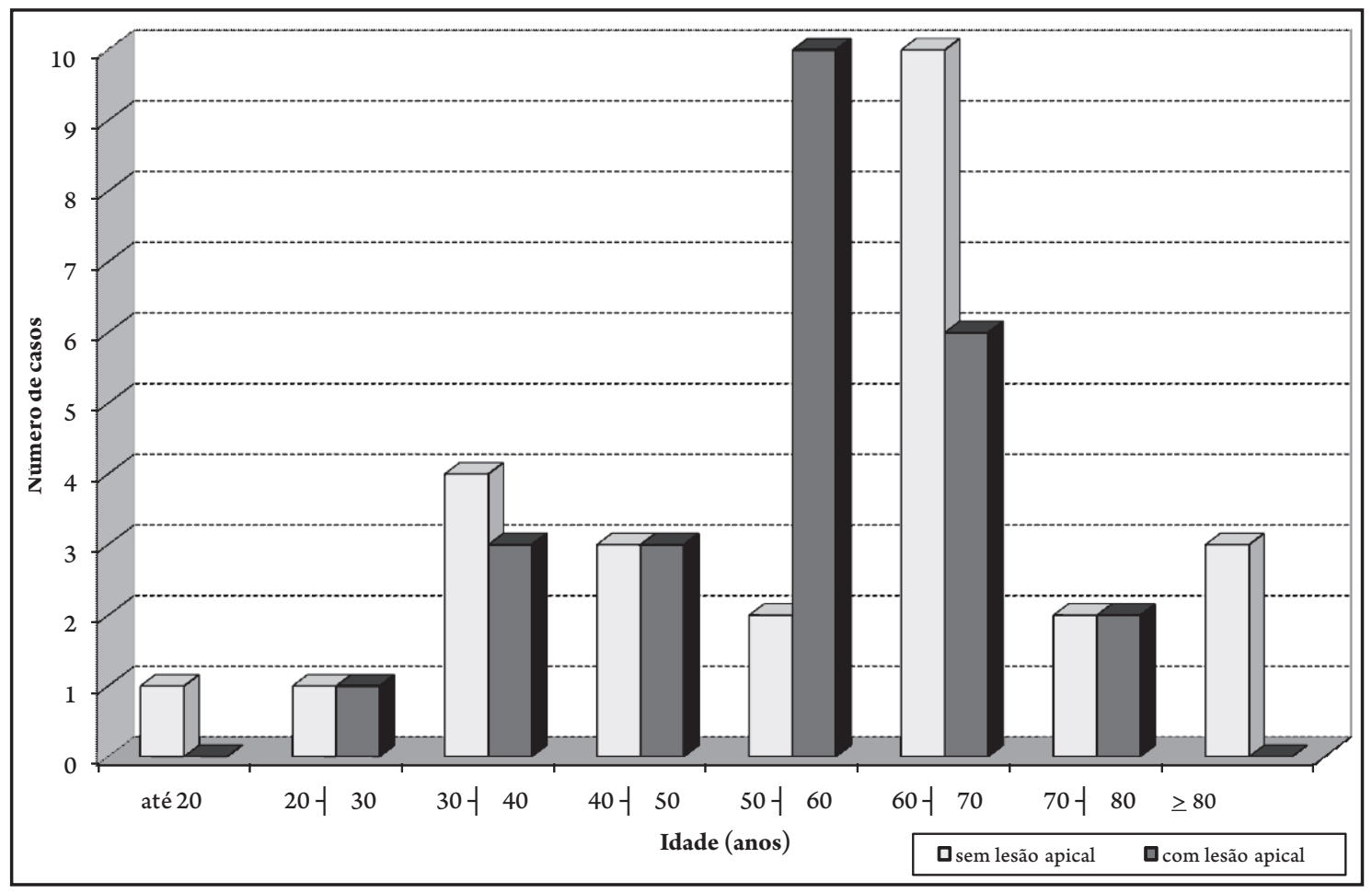

FIGURA 1 - Distribuição dos casos segundo a faixa etária e a presença (ou não) de lesão apical.

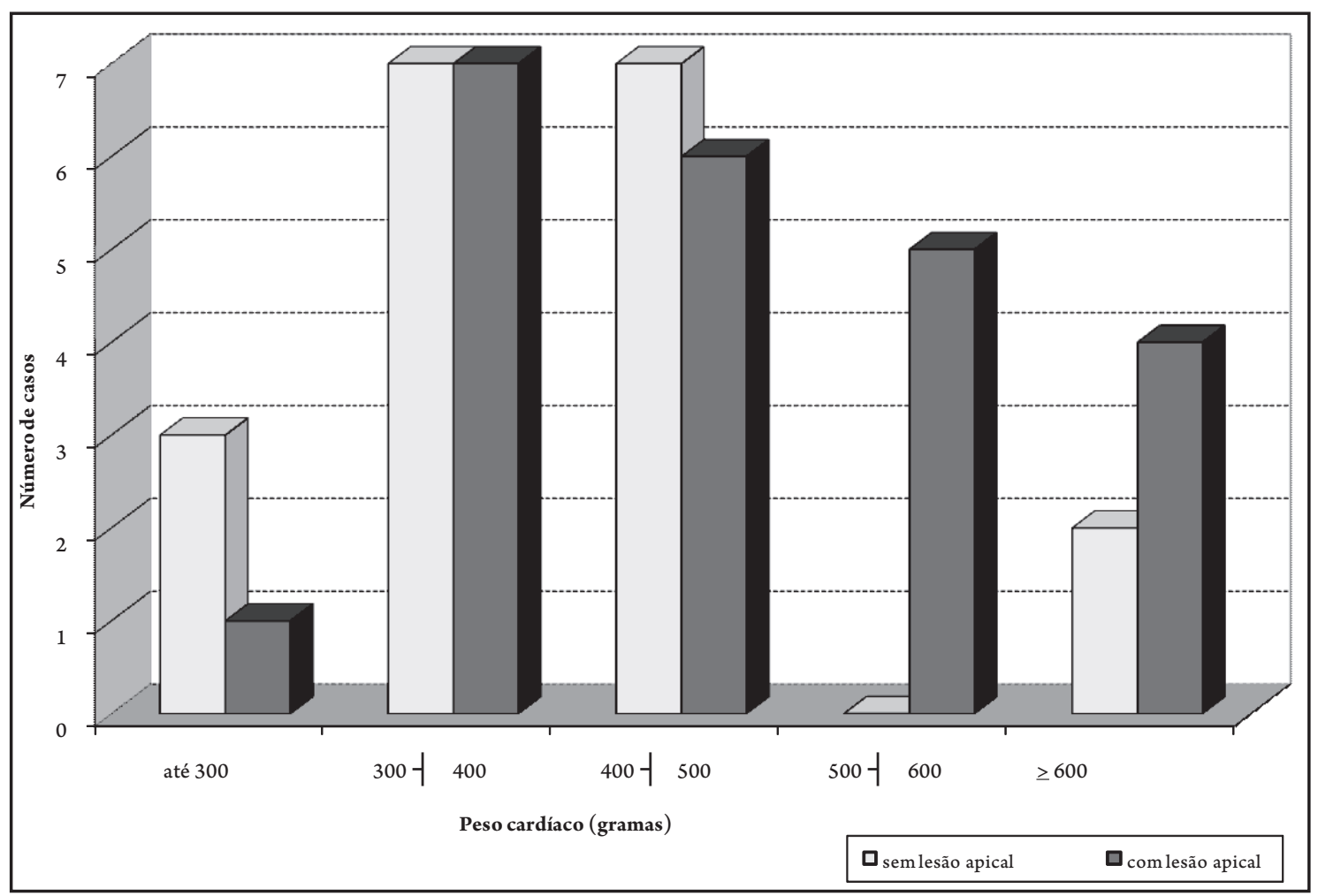

FIGURA 2 - Distribuição dos casos segundo o peso cardíaco e a presença (ou não) de lesão apical.

TABELA 1 - Distribuição das formas clínicas.

\begin{tabular}{|c|c|c|c|c|c|c|c|}
\hline \multirow[b]{3}{*}{ Formas clínicas } & \multicolumn{4}{|c|}{ Lesão apical } & \multirow{2}{*}{\multicolumn{2}{|c|}{ total }} & \multirow[b]{3}{*}{ Estatísticas } \\
\hline & \multicolumn{2}{|c|}{ não } & \multicolumn{2}{|c|}{$\operatorname{sim}$} & & & \\
\hline & $\overline{\mathrm{n}^{\mathrm{o}}}$ & $\%$ & $\overline{\mathrm{n}^{\mathrm{o}}}$ & $\%$ & $\mathrm{n}^{\mathrm{o}}$ & $\%$ & \\
\hline Miopatia & 16 & 43,2 & 21 & 56,8 & 37 & 100,0 & $\chi 2(1)=3,229 ; \mathrm{p}>0,05$ \\
\hline Batmopatia & 15 & 45,5 & 18 & 54,5 & 33 & 100,0 & $\chi^{2}(1)=1,142 ; \mathrm{p}>0,05$ \\
\hline Dromopatia & 9 & 40,9 & 13 & 59,1 & 22 & 100,0 & $\chi^{2}(1)=1,570 ; p>0,05$ \\
\hline Três formas & 6 & 33,3 & 12 & 66,7 & 18 & 100,0 & $\chi^{2}(1)=3,467 ; \mathrm{p}>0,05$ \\
\hline
\end{tabular}

TABELA 2 - Localização das tromboses.

\begin{tabular}{|c|c|c|c|c|c|c|}
\hline \multirow[b]{3}{*}{ Trombose } & \multicolumn{4}{|c|}{ Lesão apical } & & \\
\hline & \multicolumn{2}{|c|}{ não } & \multicolumn{2}{|c|}{$\operatorname{sim}$} & \multicolumn{2}{|c|}{ total } \\
\hline & $\mathrm{n}^{\mathrm{o}}$ & $\%$ & $\mathrm{n}^{\mathrm{o}}$ & $\%$ & $\mathrm{n}^{\circ}$ & $\%$ \\
\hline Não & 18 & 88,5 & 10 & 40 & 33 & 64,7 \\
\hline Sim & 8 & 11,5 & 15 & 60 & 18 & 35,3 \\
\hline Total & 26 & 100,0 & 25 & 100,0 & 51 & 100,0 \\
\hline
\end{tabular}




\section{DIscussão}

A prevalência da lesão apical apresenta grande variabilidade, dependente da característica do estudo e da tecnologia empregada para seu diagnóstico. Desta forma, encontram-se valores tão diversos quanto $40 \%$ a $77 \%$ (através da ventriculografia), $14 \%$ a $46 \%$ (pelo ecocardiograma) e $30 \%$ a $86 \%$ em avaliações necroscópicas ${ }^{12}$. A diferença nos achados está provavelmente relacionada aos distintos padrões de avaliação inerentes a cada método, sendo ainda influenciada por outros fatores, tais como precisão da aparelhagem utilizada, morfologia torácica, presença de co-morbidades pulmonares e pelo discernimento e experiência dos observadores.

Exames como a ventriculografia e a ecocardiografia avaliam a dinâmica miocárdica que, na maioria das vezes, apresenta-se alterada, não apenas em função da lesão muscular (intensidade da miocardite, da fibrose, por exemplo), mas também secundariamente, devido à presença de bloqueios de ramo, comuns na CCC. A discinesia notada nestes exames, algumas vezes não permite um diagnóstico preciso das lesões apicais como os observados em estudo anatômico puro.

À avaliação anatomopatológica macroscópica é possível observar se, na ponta do coração, há afilamento abrupto ou progressivo, característica que altera a dimensão da lesão. É possível, ainda, verificar se há fibrose ou eventual espessamento atribuível à presença de trombos (inclusive organizados e incorporados à parede ventricular) e, finalmente, se há ou não lesão coronariana, critério que permite afastar a hipótese de infarto do miocárdio cicatrizado. À microscopia óptica são obtidos dados adicionais, como intensidade da fibrose, presença de infiltrado inflamatório, afastamento dos feixes musculares e presença de trombos de pequenas dimensões.

Desta forma, justificam-se achados necroscópicos de até $86 \%$ na frequência de lesão apical. Contudo, apesar das facilidades oferecidas pelo método, é grande a variabilidade desses achados na literatura, supostamente causadas por diferenças regionais relacionadas à cepa do Trypanosoma e à própria resposta do indivíduo à agressão ${ }^{6-8}$.

No presente estudo, encontramos lesão apical em 49\% dos casos, uma frequência concordante entre as encontradas na literatura ${ }^{6-8}$. Não houve diferença significativa, entre os grupos com e sem lesão apical, em relação à idade ou ao gênero. Apesar da miopatia ter sido a alteração mais frequente nos dois grupos, notou-se pequeno predomínio desta forma nos casos com lesão apical ${ }^{2}$. Neste grupo, também foi observado discreto predomínio de lesão apical no sexo masculino sem, contudo, corroborar dados da literatura que consideram o gênero masculino e a presença de lesão apical como um fator de mau prognóstico ${ }^{13,14}$.

A média de peso cardíaco no grupo $\mathrm{A}$ foi maior em relação ao $\mathrm{B}$, porém sem relevância estatística. Esses achados confirmam os dados observados na literatura onde, da mesma forma, não foi encontrada correlação entre o peso de corações chagásicos e a presença ou ausência de lesão apical ${ }^{8,15}$.

Em ambos os grupos, observa-se relação diretamente proporcional entre um maior peso cardíaco e presença de trombose. Neste aspecto, são conhecidos os efeitos deletérios da disfunção ventricular grave na formação de trombos, principalmente se houver associação com arritmias e lesão apical ${ }^{16,17}$. Entretanto, neste estudo não houve nítida associação entre maior frequência de trombos intracavitários e lesão apical, embora tal lesão fosse sede de trombose em mais de 50\% dos casos do grupo A. Em ambos os grupos, os trombos estavam localizados principalmente no átrio direito e foram os responsáveis potenciais pelo tromboembolismo pulmonar observado.

Ainda em relação ao quadro clínico, a arritmia cardíaca esteve significativamente mais presente no grupo A, o que pode ser secundário ao predomínio de miopatia nesse grupo e pelo fato de ser a lesão apical foco potencial de arritmias.
A co-morbidade mais comum, em ambos os grupos, foi hipertensão arterial sistêmica (HAS). Embora observada com maior frequência no grupo A, onde havia maior número de casos com miopatia, não foi encontrada relevância estatística entre os grupos. O presente estudo corrobora resultados observados anteriormente pelos mesmos autores que ao avaliarem comparativamente chagásicos hipertensos e não hipertensos, não encontraram influência da hipertensão arterial sistêmica na evolução da lesão apical ${ }^{18}$.

Embora não tenha sido observada diferença significativa, o óbito atribuído à arritmia cardíaca foi maior no grupo com lesão apical, o que poderia ser atribuído tanto à maior miopatia quanto à presença da lesão apical neste grupo.

Concluímos que, apesar da lesão apical ser sede importante de trombos, ela não foi relevante para que houvesse um maior número de casos de trombose. Nesteestudo a miopatia (com aumento de peso acima de $500 \mathrm{~g}$ ) foi primordial para o aparecimento desta complicação em ambos os grupos.

\section{CONFLITO DE INTERESSE}

Os autores declaram não haver nenhum tipo de conflito de interesse no desenvolvimento do estudo.

\section{REFERÊNCIAS}

1. Lima RS, Soares MBP, Santos RR. Terapia Celular na Doença de Chagas. Rev Bras Hematol Hemoter 2009; 31(supl I):87-92.

2. Fragata-Filho AA. Tratamento etiológico da doença de Chagas. Rev Soc Cardiol Estado de São Paulo 2009; 19:2-5.

3. Almeida HOA. Lesão Vorticilar da Cardiopatia Chagásica Crônica. [Tese de Doutorado]. [Belo Horizonte]. Faculdade de Medicina da Universidade Federal de Minas Gerais; 1978.

4. Almeida EA, Manigot DA, Guariento ME, Curti HJV, Santos TCL, Bastos A, et al. A Lesão Apical: Aspectos Eletrocardiográficos. In: Cançado JR, Chuster M, editores. Cardiopatia Chagásica. Belo Horizonte: Fundação Carlos Chagas; 1985. p. 148-155.

5. Silva JLL, Teixeira MM, Pedroso ERP. A Lesão Cardíaca Vorticilar Associada à Doença de Chagas. Rev Med Minas Gerais 2003; 13:179-182.

6. Carvalhal S, Bittencourt LAK, Nogueira EA. A Lesão Apical na Cardiopatia Chagásica Crônica. Arq Bras Cardiol 1980; 35:171-177.

7. Moia B, Rosembaum MB, Hojman D. Aneurismas Ventriculares em La Miocarditis Cronica Chagasica. Rev Argent Cardiol 1955; 22:113-150.

8. Oliveira JAM. Heart Aneurysm in Chagas' Disease. Rev Inst Med Trop Sao Paulo [internet]. 1998 set-out [acessado em 8/2009]; 40:(5).Disponível em: <http:// www.scielo.br/scielo.php?pid=S0036-46651998000500007\&script $=$ sci arttext\&tlng=en $>$.

9. Curti HJV, Sanches PCR, Bittencourt LAK, Carvalhal SS. Revisão da classificação anátomo-clínica da doença de Chagas. Arq Bras Cardiol 1979; 33:277-281.

10. Werkema MCC. Ferramentas Estatísticas para o Gerenciamento de Processos. Belo Horizonte: Fundação Cristiano Otonni, Escola de Engenharia, Universidade Federal de Minas Gerais; 1995.

11. Levin J. Estatística Aplicada em Ciências Humanas. São Paulo: Harper e Row do Brasil Ltda; 1978.

12. Borges-Pereira J, Xavier SS, Souza AS, CastroJAF, Zauza PL, Coura JR. Prevalência de Aneurismas do Ventrículo Esquerdo em Pacientes Chagásicos Crônicos de duas Áreas do Estado do Piauí. Rev Soc Bras Med Trop 2007; 40:521-526.

13. Mady C, Nacruth R. História Natural da Cardiopatia Chagásica Crônica: fatores prognósticos. Rev Soc Cardiol Estado de Sao Paulo 1994; 4:124-129.

14. Pereira-Barretto AC, Arteaga E, Mady C, Ianni BM, Bellotti G, Pileggi F. Sexo Masculino: Fator Prognóstico na Doença de Chagas. Arq Bras Cardiol 1993; 60:225-227.

15. Almeida HO. Hipóxia como Fator Patogenético da Lesão Apical em Chagásicos Crônicos. Rev Goiana Med 1986; 32:129-139.

16. Braga JC, Labrunie A, Villaça F. Thromboembolism in Chronic Chagas' Heart Disease. São Paulo Med J 1995; 113:862-866

17. Mady C, Nacruth R. Natural History of Chronic Chagas' Disease: Prognosis Factors. São Paulo Med J 1995; 113:791-796.

18. Gurgel CBFM, Almeida EA. Freqüência da Hipertensão Arterial em Chagásicos Crônicos e sua Repercussão no Coração: Estudo Clínico e Anatomopatológico. Arq Bras Cardiol 2007; 89:191-200. 